\title{
La stratégie narrative dans "Les Catilinaires" et "Péplum" d'Amélie Nothomb
}

\section{Par \\ Dr.Alaaedin Baheidin Alaaedin*}

alaabahi364@gmail.com

\section{Résumé}

Amélie Nothomb est née au Japon en (1967). Le Japon se montre dans l'œuvre de Nothomb comme un objet romanesque. L'influence littéraire du Japon dans son œuvre était très évident dans ses récits fictionnels et dans ses romans autobiographiques.

Dans notre étude, nous allons mettre la lumière sur la technique de l'écriture de Nothomb et le rôle qu'elle joue dans les relations humaines, ainsi que les thèmes prédominants qui hantent l'esprit de Nothomb. Le roman "Les Catilinaires" sera la base de cette partie de notre étude. Dans la seconde partie de notre travail, nous allons aborder les fonctions diverses de la narration de Nothomb à travers le roman "Péplum" comme: la fonction dramatique, la fonction psychologique et la fonction descriptive.

\section{Mots clés: La stratégie - La narration- Amélie Nothomb.}

\section{Introduction}

Ecrivaine belge d'expression française, Amélie Nothomb est née au Japon en (1967). Son nom "Nothomb" est un des noms les plus connus de Belgique. Son père Patrick Nothomb était un diplomate depuis (1964). Or, Amélie le suit dans ses voyages partout à l'étranger. Puis ces voyages ont formé et dessiné la personnalité littéraire de cette romancière.

* Professeur adjoint à la faculté des Lettres - Université du Sud de la vallée 
Celle-ci connaît la Belgique qu'à l'âge de dix-sept ans, quand elle a étudié la philologie à l'Université libre de Bruxelles. Dès ce moment, elle a commencé à écrire dans sa chambre en souffrant de la solitude qu'elle éprouve et qu'elle nous avons montré plus tard dans son œuvre.

Depuis son premier roman « Amélie Nothomb a occupé une place privilégiée dans la littérature française. Sa biographie comprend plusieurs œuvres romanesques. Elle a été récompensée par le Grand prix du roman de l'Académie française en (1999) pour son œuvre "Stupeur et tremblements" (1999). Amélie Nothomb a pu imposer un prestige littéraire dès l'apparition de son premier roman.

En (1988), Nothomb retourne au Japon, dorénavant, elle jouit d'une célébrité sur la scène littéraire internationale. Et l'écriture a donné un sens à la vie de cette romancière belge.

Le Japon se montre dans l'œuvre de Nothomb comme un objet romanesque. Même on peut trouver dans ses romans une confrontation entre le culture orientale et la culture occidentale.

Dans notre étude qui sera divisée en cinq points essentiels, nous allons mettre la lumière sur la technique de l'écriture de Nothomb et le rôle qu'elle joue dans les relations humaines, ainsi que les thèmes prédominants qui hantent l'esprit de Nothomb. Le roman "Les Catilinaires", un des deux romans sera à la base de notre étude, où dans ce roman, la solitude à deux, tel était le rêve d'Emile et de Juliette. Une maison au fond de la forêt pour y 
terminer leurs derniers jours. Un autre voisin nommé Palamède Bernardin est venu se présenter, puis a pris l'habitude d'aller chez eux chaque après-midi sans dire un mot. Et cette présence absurde va devenir de plus en plus dérangeante et les visites deviennent un enfer. C'est une comédie très noire, d'une lucidité bizarre et

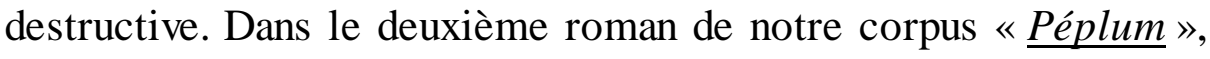
c'est un roman de la science-fiction, satire, finesse psychologique d'un affrontement verbal où chacun cherche la faille de l'autre : dans ce mélange, nous trouvons l'insolence et le style distingué de cette romancière qui la placent au premier rang et parmi l'élite des écrivains de sa génération. Nous allons aborder les fonctions diverses de la narration de Nothomb à travers ce roman, telles la fonction dramatique, la fonction psychologique et la fonction descriptives.

\section{I- Caractéristiques de l'écriture nothombienne}

"Mon écriture, c'est une tentative de définition du monde et du langage, très souvent avec l'œil enfantin qui essaie de nommer les choses, de leur donner le nom juste." (Interview, 12 mars 2009).

L'importance majeure de l'écriture nothombienne réside dans le rôle que cette écriture se propose de fortifier les relations humaines, Nothomb a réussi à réaliser ce but à travers ses livres autobiographiques comme: Métaphysique des Tubes (2000), Le Sabotage Amoureux (1993), Stupeurs et Tremblement (1999), Une forme de Vie (2010)... Le besoin d'écriture l'a aidée à dépasser la 
solitude dont elle avait souffert après son retour en Europe. La cause principale de cette solitude retourne aux souffrances de l'abandon en Belgique et au Japon.

Les racines de l'écriture de Nothomb sont enracinées dans la culture japonaise, ainsi que dans l'écriture européenne. Nous remarquons le mythe japonais à travers quelques romans de Nothomb comme: "Métaphysique des tubes" (2000), "Stupeur et tremblements" (1999), "Les Catilinaires" (1996) et "Ni d'Eve ni d'Adam" (2007) parce que: "Tout ceux qui ont un jour entendu parler d'Amélie Nothomb savent qu'elle est totalement, tendrement, voire tragiquement imprégnée par le Japon où elle a passé la première partie de son enfance." (Zumkir, 2003: 95)

Il est à remarquer que Nothomb, depuis (1992) a publié régulièrement un livre à chaque année, un succès incroyable en tout cas: "On a dit qu'elle était "surdouée" et "brillante",..." (Vigeant, 1997: 162). L'autobiographie et la fiction forment presque la ligne essentielle de la production littéraire de Nothomb, pour elle: "L'écriture est une activité vitale qui l'aide à lutter contre son ennemi intérieur qui ne cesse de lui répéter ses limites." (Gingras, 2003: 37). Evelyne Wilwerth affirme la même chose dans son article intitulé "Amélie Nothomb sous le signe du cinglant" dans "La Revue Générale" en disant que: "Sa vitalité est encore renforcée par le dialogue en tant que confrontation, la plus directe des relations humaines." (Wilwerth, La Revue Générale, 1997).

(La stratégie narrative ...) Dr. Alaaedin Baheidin Alaaedin 
De son côté, Jean Decker s'appuie surtout sur le mode de la narration de chacun des romans en disant que: "Ceux qui font une place royale au dialogue... il s'agit d'Hygiène de l'assassin, des Catilinaires..." (De Decker, 2003: xii). Nous remarquons que Nothomb se raconte en utilisant le pronom "je" dans cinq de ses romans, tandis que "Les Catilinaires" et "Attentat" (1997) sont également narrés à la première personne, mais le narrateur est fictif: "nous verrons que le "je" nothombien est un "je" mystérieux dont la définition a besoin d'être plus ouverte car tous ces romans de la première personne de singulier ne sont pas exclusivement autobiographiques." (Delangue, 2014: 130).

Mais à première vue, l'écriture de Nothomb était concentrée sur quelques thèmes qui l'obsèdent toute sa vie, le japonisme, l'autre et la relation avec lui, la mort, l'ennemi qui est en nous, le corps avec sa beauté et ses métamorphoses, le poids des mots et leur influence sur les autres, l'angoisse de l'abandon....etc. Ces sujets peuvent plonger plus loin dans les rapports humains, car la littérature pour l'écrivaine n'est qu'un moyen de se rapprocher les uns des autres:

"... avec Amélie Nothomb, on est de plain-pied dans la littérature en actes, dans l'urgence d'écrire qui impose une perpétuelle- écriture..." (David, 2013, 15).

Amélie Nothomb a traité dans ses romans plusieurs sujets qui forment un réseau, à travers l'ensemble de ses ouvrages, nous pouvons constater les techniques différentes d'écriture qui 
caractérisent notre auteure, son écriture fluide et percutante, elle indique également que son écriture consiste quelquefois à gratter pour aller chercher la matière. D'autre part, la musique a une grande influence sur elle en lui donnant une vision musicale de son écriture. Parmi ces thèmes traités dans ses romans, nous avons des sujets déjà connus de la littérature traditionnelle, comme la mort, la solitude et d'autres sont distingués par l'innovation comme l'amitié.

\section{1) La Solitude}

Le thème de la solitude est presque enraciné dans toute l'œuvre de Nothomb. Ce thème retourne à l'abandon souffert en Belgique et au Japon. La hantise du sujet de la solitude n'a pas de notion mauvaise ou néfaste, mais cette solitude est à la fois désirée et horrible. Dans "Les Catilinaires", nous apercevons par exemple, la solitude choisie qui offre la tranquillité tellement cherchée en refusant l'existence humaine, nous pouvons considérer que cette tranquillité n'est qu'une clé pour une vie stable et calme. Dans ce roman, l'auteure essaie de mettre en valeur le désir à la liberté, qui se caractérise dans le besoin de l'être de s'enfuir du monde:

"Mon proverbe favori a toujours été: pour vivre heureux, vivons, cachés." (Nothomb, Les Catilinaires: 15).

Un autre rôle de la solitude dans l'œuvre romanesque de Nothomb, la solitude est une chose indispensable pour les contemplateurs pour arriver à comprendre l'autre et se 
comprendre soi-même comme dans son roman "Stupeur et tremblements". Il est à remarquer que la place qui convient à cette contemplation dans ce roman, est les toilettes qui: "sont un endroit propice à la méditation. Pour moi qui y étais devenu carmélite, ce fut l'occasion de réfléchir." (Nothomb, Stupeur et tremblements: 162).

Egalement, au Japon, l'entreprise de Yumimoto, c'est la compagnie où à l'âge adulte, Nothomb y retourne pour un contrat, afin d'y travailler et d'y vivre comme une japonaise en tant qu'employé, où cette place dans « $\underline{\text { Stupeur et tremblements }}$ » ne représente qu'un espace clos qui ne contient que quelques personnages qui sont devenus comme de véritables bourreaux à la Fubuki, la même chose se passe entre Bernardin et les autres personnages dans «Les Catilinaires $»$ où se manifeste l'impuissance de coexister avec les autres, l'essai d'expliquer et de comprendre les relations personnelles pour son entourage nous mènent certainement à l'idée de J-P. Sartre exprimée dans son "uis clos" et qui s'accorde avec l'idée finale de Nothomb dans son œuvre, quand Sartre a déclaré que l'Enfer, c'est les Autres. Amélie Nothomb a donné une interprétation pour cette notion de la solitude, ainsi que pour les rapports entre les personnages en disant que:

"Vous avez raison de mentionner Sartre... l'enfer c'est les Autres, je suis d'accord, mais l'enfer c'est soi aussi. Soi, soi-même, vécu comme un Autre. Nous sommes, nous-mêmes,

(La stratégie narrative ...) Dr. Alaaedin Baheidin Alaaedin 
un Autre. L'enfer c'est aussi soi. Donc, où se situer de l'Autre, mais de soi-même? Soi-même, c'est ça, l'enfer." (Interview avec l'auteure, 22 mars, 2010).

\section{2- Le Corps}

Le corps humain représente un thème essentiel dans presque toute l'œuvre d'Amélie Nothomb: "Le corps est révélateur d'une identité problématique." (Narjoux, 2004: 43). Amélie Nothomb nous a présenté le corps humain avec ses deux facettes : le corps beau et le corps difforme. Le premier est celui de mademoiselle Fubuki dans «Stupeur et tremblements », le second est celui de Bernardin dans «Les Catilinaires». Ahmad Nusrat a donné la raison pour laquelle Nothomb a donné cette représentation contradictoire, en disant que: "s'intéresser à la beauté, c'est forcément s'intéresser à son contraire." (Nusrat, 2001). En s'intéressant à Fubuki; elle met en valeur la contemplation et surtout la fascination. Nous remarquons que Nothomb s'intéresse à jouer avec les périodes différentes de lavie : la jeunesse, la vieillesse....Cette structure est fréquente chez elle:

"... Notomb construit l'univers thématique de ses ouvrages sur les dualités fortes: la jeunesse et la vieillesse... Le thème de pur et impur constitue un de ses principaux questionnements." (Amanieux, 2005: 44-45).

Les impressions et les pensées de Nothomb sur la beauté ou la difformité se manifeste clairement dans ses romans et 
surtout dans "Les Catilinaires": "L'enjeu était important: monsieur Bernardin possédait-il notions du beau et du laid." (Nothomb, Les Catilinaires: 30). Dans ce roman, la moquerie se montre sur la langue de Mme Bernardin dans les répliques qui sont bien nombreuses dans l'œuvre (une masse de chair, un tentacule de gras, ça, cette chose...etc.). C'est un essai de formuler les choses strictes et contradictoires, le réel qui se contredit avec la réalité, qui vise à montrer la beauté japonaise et le corps japonais qui se caractérise par la minceur à Fubuki, car: "la maigreur hautaine (inspire) le respect.", tandis que Palamède Bernardin s'exprime en disant: "Vous mangez tant et vous restez maigres!" (Nothomb, Les Catilinaires: 72).

Donc, le respect du corps mince et l'ironie du corps gras sont les éléments essentiels que l'auteure met souvent au cœur de son œuvre en parlant de ses personnages.

D'autre part, Nothomb donne une grande importance au sujet de l'alimentation qui est en relation avec le corps humain, cela se manifeste dans presque la plupart de ses œuvres.

Le sujet de la maigreur a hanté Nothomb qui ne cesse de revenir à ce sujet d'une façon répétée, car elle est souvent entourée de personnages minces lors de ses voyages.

\section{3- La Mort}

Amélie Nothomb était occupée par la question de la mort dès son enfance. Au cours de ses œuvres, nous remarquons l'utilisation des expressions qui font allusion au sujet de la mort.

(La stratégie narrative ...) Dr. Alaaedin Baheidin Alaaedin 
Nous avons dans l'œuvre de Nothomb deux genres de mort, la mort réelle représentée par l'assassinat de $\mathrm{M}$. Bernardin, et la mort imaginaire concrétisée dans le roman "Les Catilinaires". Ce genre de mort consiste à mettre le front dans un trou vitré du quatrième étage de l'entreprise Yumimoto et à se laisser tomber fictivement, à se laisser tomber dans le chaos ou dans le vide. C'est une manière pour arriver à l'état contemplatif ou arriver à poser des questions essentielles sur l'existence et son importance pour nous et par conséquent, nous arrivons à une décision: "Une autre hypothèse était la solution de toute existence humaine: la mort." (Notomb, Les Catilinaires: 88).

Donc, nous pouvons dire, que l'auteure avait très tôt le désir de faire une rencontre avec la mort et cela se manifeste quand elle relate son histoire lorsqu'elle s'est presque noyée dans la mer ou de son essai de se suicider dans "Métaphysique des tubes" (2000), ces tentatives lui font apprendre de n'avoir peur de la mort ou d'être consciente dans des cas très proches de la mort.

En arrivant à l'état de n'avoir pas peur de la mort, cela convient avec la notion japonaise qui était basée sur cette conception que chacun a son droit de se suicider, comme un acte d'honneur pour les japonais.

\section{II- Les techniques langagières de Nothomb}

\section{1) Pouvoir du langage}

Chez Amélie Nothomb, le langage représente le véritable plaisir grâce à son pouvoir, c'est pourquoi: "elle cherche à découvrir le langage qui seul réunit pouvoir, savoir et vouloir." (Amanieux, 2005: 30). L'importance du langage et de la parole se manifeste très clairement dans son œuvre. Cette importance

(La stratégie narrative ...) Dr. Alaaedin Baheidin Alaaedin 
donnée à la parole est incontestable car elle nous aide à comprendre et à éclaircir les problématiques du sujet.

Avec la parole, Nothomb se trouve face à un problème: la contradiction qui se trouve entre la conscience de l'Occident et celle de l'Orient. En Occident, les hommes sont prêts à faire un dialogue et écouter l'autre, tandis que en Orient, on choisit le silence, parce que, quand on parle, il y a toujours des risques qui rendent les situations plus graves.

D'autre part, le langage représente le stock inépuisable des mots pour l'auteure. Ce qui donne la liberté à son imagination créative ou comme l'a précisé l'exemple suivant: "Il y a des dératiseurs, il n'y a pas de dévoisineurs." (Nothomb, Les Catilinaires: 78). Pour Nothomb, le langage a des pouvoirs très dangereux, c'est pourquoi, nous pouvons dire que la pensée est impossible sans langage car il a son influence magique et mystique sur les autres. Nous avons besoin du langage pour sortir et transmettre nos pensées aux autres et nous ne pouvons pas réaliser cela sans le langage qui est capable de faire la transmission de notre intériorité. Dans "Les Catilinaires", Nothomb a affronté l'ennemi par la parole en se référant à la puissance oratoire.

Il est à remarquer que l'auteure a essayé de montrer l'importance d'utiliser le prénom de ses personnages, soit des personnages fictifs ou réels. Pour elle, le prénom est un réservoir d'informations qui découvre la véritable identité pour chacun, comme Claire, l'amie de la famille Hazel dans "Les Catilinaires" qui affirme que chaque personnage: "portait son prénom à merveille: il émanait d'elle une lumière qui captait le regard. Elle faisait partie de ces êtres d'exception dont la simple présence suffit à rendre heureux." (Nothomb, Les Catilinaires: 92).

Nous remarquons qu'il y a une bonne opposition dans le cas de monsieur Bernardin où sa présence suffit à apporter le

(La stratégie narrative ...) Dr. Alaaedin Baheidin Alaaedin 
malheur, ainsi que, nous trouvons la même chose dans le cas de: "Palamède et Bernadette Bernardin. Un prénom étrange à un prénom banal mais itératif. C'est merveilleux." (Nothomb, Les Catilinaires: 63).

En parlant du langage nothombien, nous ne pouvons pas négliger les effets de ce langage qui donnent à chaque fois un style tout à fait divers, une fois, nous trouvons un style absurde, d'autres fois, comique ou critique.

Premièrement, nous abordons le comique qui provoque le sourire et le rire, Cécile Narjoux explique cet effet du langage en citant que: "toutes les formes d'expression qui visent à provoquer le rire et le sourire... Le rire- ou le sourire- naît d'une contradiction, d'une situation qui crée un écart soudain par rapport à ce qui est attendu." (Narjoux, 2004: 73). Amélie Nothomb, pour réaliser cet effet comique, elle a insisté sur le sens contraire de ce que nous énonçons.

Le comique chez Nothomb est lié solidement à l'ironie. Elle ironise quelquefois la situation, elle ne cherche pas le drôle, mais elle écrit avec une certaine distance. Cette ironie sert à créer un genre de désaccord, d'opposition dans les idées. Donc, l'ironie dépend à dire le contraire de ce à quoi nous réfléchissons. Par exemple, dans "Les Catilinaires", pour montrer le mécontentement des Hazel après les visites consécutives de $\mathrm{M}$. Bernardin et pour lui dire que sa présence n'est pas désirée, l'ironie se montre dans son intégrité et sa totalité comme dans l'extrait suivant:

"Oh! Quelle surprise! M'exclamai-je en découvrant notre tortionnaire... Savez-vous que vous m'avez sauvé la vie, hier? Qu'est-ce qui vous ferait plaisir... De la tarte aux pommes?... Du pudding écossais? Un miroir au cassis? Des éclairs au chocolat?" (Nothomb, Les Catilinaires: 64-74). 
Quant au deuxième effet du langage nothombien, c'est l'absurde. Nous pouvons considérer l'absurde un degré très élevé du comique: "Est absurde, ce qui est contraire à la raison, au sens commun." (Narjoux, 2004: 76). L'absurde paraît dans l'œuvre de Nothomb dans la stupidité répétitive et dans la fréquence permanente que nous pouvons considérer comme une certaine critique de la société.

\section{2) Réalité du langage nothombien}

Nous remarquons que les personnages dans les romans de Nothomb sont tout proches de l'écrivaine et surtout quand il s'agit d'écrire sur soi-même, en ce cas, les personnages ressemblent à l'auteure et surtout en ce qui concerne la fiction. L'existence du soi nous a imposé la question de la distance entre la fiction et l'autobiographie. Donc, nous pouvons dire que Nothomb se montre dans ses ouvrages qui portent l'aspect autobiographique:

"... chacun des personnages ou des groupes présents peut donc lui être miroir... et apparaître comme une définition... d'ellemême." (Narjoux, 2004: 38).

La distance qui sépare la réalité de la fiction peut être limitée avec une grande certitude, c'est pourquoi, il faut que nous soyons très attentifs, en ce qui concerne les ouvrages autobiographiques. Dans l'écriture autobiographique, c'est la vérité, qui est la plus importante chez l'écrivaine en exprimant ses émotions, mais n'a aucune importance à l'égard des événements. L'auteure subit la même position et les mêmes sentiments à l'égard de sa propre autobiographie. Mais la véritable problématique qui affronte Nothomb, c'est comment construire 
une identité, notre écrivaine a parlé des identités diverses dans ses ouvrages. Dans "Les Catilinaires", Nothomb a proposé le problème identitaire d'une façon claire en disant:

" Je ne sais plus rien de moi." (Nothomb, Les Catilinaires: 151).

\section{III- Du roman classique au roman dialogué}

"Dans les romans, la part dite dialogue est l'expression de la paresse et de la routine: Les personnages parlent pour mettre des blancs dans une page, et par imitation de la vie où il n'y a pas de récits, mais des conversations..."

(Blanchot, 1971: 225).

Dans les romans conventionnels, nous pouvons raconter une histoire sans donner la parole aux personnages, parce que le dialogue dans ce roman n'est qu'une ruse: "car le grand alchimiste de leurs discours, c'est l'auteure, ce locuteur suprême se sert d'eux comme d'autant de voix dociles, à qui il fait dire ce qu'il veut..." (Berthelot, 2001: 1). De son côté, Vivienne G. Mylne, dans son livre "Le dialogue dans le roman français de Sorel à Sarraute" a précisé que: "Si l'ouvrage entier est fait de conversations présentées sans verbes déclaratifs, on peut le classer tout suite, semble-t-il parmi les romans dialogués." (Mylne, 1994: 171).

D'autre part, nous pouvons proposer une autre définition qui concerne le roman dialogué où Marie-Hélène Boblet dans son livre intitulé "Le roman dialogué après 1950. Poétique de l'hybridité", a estimé le roman dialogué comme: "un texte de

(La stratégie narrative ...) Dr. Alaaedin Baheidin Alaaedin 
matière romanesque, envahi par le dialogue au point que la proportion entre le narratif et le discursif..." (Boblet, 2003: 13). Mais, puisque le roman dialogué reste un roman comme les autres romans, nous trouvons que les définitions précédentes du roman dialogué ne s'accordent pas avec la notion traditionnelle du roman, car le roman n'est qu'un récit, il nous offre des personnages qui jouent leurs rôles dans un certain temps et dans un certain espace. Celui qui relate ce récit est le narrateur, c'est la voix qui prend en charge l'histoire. Donc, nous pouvons dire que le dialogue dans les romans conventionnels dépend toujours de la narration:

"Les conversations sont rarement importées d'un seul tenant dans le récit." (Durrer, in Pratiques, 1990: 40).

A l'instar du dialogue dans les pièces de théâtre, le dialogue dans les romans qui est soumis à la narration offre également quelques effets par rapport au dialogue du théâtre. Donc, nous apercevons que le dialogue dans le roman conversationnel n'est qu'une ruse au service du narrateur:

"il n'a pas besoin de présenter les personnages, de rapporter le passé- tout cela est fait généralement par le récit-il peut rester allusif..." (Mitterand, 1985: 142).

Dans le roman conversationnel, le dialogue peut occuper plusieurs fonctions en cas d'absence de narrateur, de construire les personnages, de développer l'action et les thèmes, de présenter des idées et finalement de raconter le récit. Comme le cas dans le 
dialogue de théâtre, le dialogue dans le roman peut occuper la fonction d'exposition, ainsi qu'il peut prendre en charge le rôle de la caractérisation, qu'il peut montrer expliquer les caractères, ainsi que les objectifs et les émotions des personnages dans le roman. Mais, le rôle le plus important que joue le dialogue dans le roman réside dans l'action, il s'intègre dans l'évolution dramatique et développe l'intrigue.

Mais, nous remarquons que ce qui manque au roman dialogué ou conversationnel, qu'il ne relate rien et qu'il ne s'y passe rien, c'est le cas de dialogue d'idées dans le théâtre, dont sa structure n'est: "ni narrative ni dramatique mais conceptuelle." (Guellouz, 1992: 62).

Dans le roman de « $\underline{\text { Péplum }}$, nous remarquons que l'intrigue se place sous une forme de duel. Dans ce roman, nous avons deux dialogues avec un petit nombre d'interlocuteurs. Le roman s'ouvre sur un dialogue entre une femme qui parle de Pompéi qui serait la création des archéologues du futur et un homme qui rejette sa parole :

"- Cherchez à qui le crime profite.

L'ensevelissement de Pompéi sous les cendres du Vésuve, en 79 après Jésus-Christ...A votre avis, qui a fait le coup?

-Pas mal, comme sophisme.

-Et sice 'était pas un?

-Que voulez-vous dire?

-Cela ne vous a jamais paru bizarre? Il y avait des milliers villes à détruire. » (Nothomb, Péplum : 5) 
Un autre passage narratif à la première personne du singulier, dans lequel, la femme narre comment elle se réveillée à l'hôpital en se trouvant dans le futur :

"A mon réveil, l'hôpital était méconnaissable.

Ma chambre avait des dimensions s'une salle de bal....

La distance qui me séparait du sol semblait de deux mètres. J'hésite à sauter....

Tant pis. Je n'allais pas demander l'aide des infermières pour si peu. Je me dirigai vers la porte. Je l'ouvris et je tombai dans le vide.» (Nothomb, Péplum : 11)

Ensuite, le second dialogue commence avec duel verbal qui occupe la majorité des pages du roman. La narratrice se trouve dans l'année 2580 avec Celsius, un savant. Celui-ci finit par la renvoyer à son époque en espérant qu'elle peut lui assurer l'éternité en lui consacrant un livre.

Ainsi, le dialogue dans «Péplum» s’intéresse par la situation essentielle et la série de paroles qui changent cette situation. Pendant ce long dialogue, les cours passages narratifs montrent quelques silences :

"Je hais le vingt-sixième siècle....Si vous essayez de me le dire, je me bouche les oreilles.

-Je me bouchai les oreilles. Je voyais les lèvres de Celsius qui continuaient à remuer....» (Nothomb, Péplum : 27)

\section{IV- La narration dialoguée dans le roman "Péplum"}

"Amélie Nothomb se dit "dialoguiste" et non romancière. Elle maîtrise parfaitement cette 
technique, avec un plaisir non dissimulé."

(Kobialka, 2006: 10).

A première vue, le roman "Péplum" écrit en (1996) ne semble pas raconté par un narrateur, mais par l'ensemble des paroles échangées par les personnages. Il est à remarquer que le roman dialogué est avant tout, un roman qui se distingue par son procédé narratif et par sa forme conversationnelle.

Dans le roman "Péplum", la parole des personnages est riche, le roman se distingue par la dramatisation, où Nothomb a réussi à mettre en valeur des échanges verbaux où la parole fait développer l'action et l'intrigue. La parole a représenté des fonctions différentes dans le roman. L'étude des fonctions et des rôles exercés dans le roman nous a montré que la parole des personnages y remplit, parmi ces fonctions: la fonction descriptive, la fonction dramatique et la fonction psychologique qui seront analysées plus tard dans notre travail:

"Les textes d'Amélie Nothomb étant reconnus comme appartenant à une "bonne" littérature à la fois accessible, cultivée et dotée de qualités esthétiques..." (Saunier, 2015: 124).

Le narrateur dans les romans dialogués de Nothomb raconte l'histoire en exposant la situation essentielle et donne le dénouement à la fin du roman. Mais dans "Péplum", la narration y est impliquée, elle est concrétisée dans la femme de la première conversation, ainsi qu'elle sera le personnage principal de la seconde conversation: 
"Celsius avait raison: je n'ai pu faire autrement que d'écrire un livre sur lui. Ce ne fut pas facile. Il a fallu que je retranscrive, de mémoire, notre long échange..." (Nothomb, Péplum: 152).

Dans cet extrait, la narratrice joue son rôle d'affirmation en essayant de convaincre le lecteur que ce dialogue se retrouve effectivement. Donc, les narrateurs dans les romans de Nothomb restent des éléments essentiels dans le roman, ces narrateurs permettent d'achever le discours des personnages. Dans "Péplum", le narrateur affirme la sincérité des conversations, il semble que la narration est liée parfaitement aux dialogues qui concrétisent non seulement l'intrigue dramatique de ces romans, mais aussi qui peuvent endosser les rôles traditionnels qui concernent le narrateur.

Donc, nous pouvons dire que le dialogue occupe généralement une place très importante dans les romans de Nothomb. Dans notre roman "Péplum" qui est considéré comme un roman dialogué par excellence, la situation initiale informe le lecteur sur les personnages et leurs relations afin de lui faciliter la compréhension correcte de l'action.

Dans "Péplum", nous remarquons deux dialogues, le second occupe plus de 140 pages où nous n' apercevons que de courtes interventions de la part des narrateurs. Le roman n'est qu'un récit dialogué qui raconte une histoire fictive, c'est pourquoi, il s'accorde avec la définition du roman dialogué. 
Le dialogue dans "Péplum" vient à l'instar du dialogue d'idées. Le roman est raconté par des paroles qui le forme, grâce aux échanges verbaux qui portent le caractère polémique et didactique et qui permettent au dialogue de remplir les fonctions romanesques.

Egalement, dans les romans conversationnels ou dialogués de Nothomb, nous trouvons que les nœuds nous mènent à une situation conversationnelle, c'est pourquoi, nous remarquons dans "Péplum" que la conversation n'est qu'une succession de paroles qui poussent le roman vers un dénouement.

D'autre part, l'intrigue dans "Péplum" se manifeste comme un signe du conflit, car le dialogue représente: "le développement, la mise en forme de deux positions discursives confrontées ou affrontées." (Ubersfield, 1993: 263).

Donc, le dialogue est généralement soumis à la narration qui demeure dominante. Dans son article "Dialogue et littéralité romanesque", Henri Mitterand se demande ce qui: "induit, dans la composition d'un roman, dans l'agencement de ses éléments et de ses arguments, la possibilité et la nécessité du dialogue." "Mitterand, Le dialogue, 1985: 143).

D'autre part, nous apercevons que les personnages dans les romans conversationnels nothombiens s'affrontent face à face au cours d'un duel verbal: "...les protagonistes ont une forte tête et savent user des ressources langagières pour plaquer l'autre... chaque affrontement est une bataille de nerfs qui s'engage."

(La stratégie narrative ...) Dr. Alaaedin Baheidin Alaaedin 
(Gingras, Erudit, 2003: 37). C'est pourquoi, il est important de définir leur identité, ainsi que de comprendre la relation qui les rassemblent car: "Chaque moment de l'action constitue une situation conflictuelle où les personnages se poursuivent, s'allient ou s'affrontent." (Bourneuf et Ouellet, 1989: 160).

En bref, la narration se développe pour se transformer en un récit sur le mode dramatique. A ce moment, le personnage s'efface également et se métamorphose à un corps dénué des sentiments et de conscience:

"En effaçant les dernières remarques de l'instance narrative et en donnant d'emblée la parole au personnage." "Genette, 1972: 193).

\section{V- Les fonctions narratives dans "Péplum"}

\section{1) La fonction descriptive}

Le rôle principal de la description réside essentiellement dans la représentation des personnages, des actions et des péripéties en formant un cadre, dans lequel, les actions occupent place. Cette fixation du récit dans un cadre spatio-temporel est importante, c'est pourquoi la description joue un rôle essentiel dans le roman, elle permet au lecteur de plonger dans le monde réel ou fictif du récit qu'elle construit.

"Péplum" représente le roman qui a le moins de paragraphes narratifs. La parole des personnages crée un cadre, dans lequel, l'échange verbal prend place. Dans ce roman, nous

(La stratégie narrative ...) Dr. Alaaedin Baheidin Alaaedin 
trouvons que le premier dialogue ne porte pas d'informations nécessaires sur le temps et le lieu des événements. Tandis que, dans le second dialogue, nous trouvons les répliques qui forment le cadre: ces répliques découvrent le temps, le lieu et la raison du dialogue.

Dans "Péplum", l'action se passe chez l'un des interlocuteurs, qui vit dans une basilique, le 27 mai 2580: l'action de la narration aurait eu lieu 585 années plus tôt, soit le 8 mai 1995. En plus, nous savons que l'homme s'appelle Celsius, et que la femme est nue et sera vêtue d'un péplum, nous allons apprendre également que Celsius porte un hologramme:

"- Un péplum?

- C'est cela ou rien.

- Le péplum est revenu à la mode, en $2580 ?$

- Non. C'est un costume de théâtre.

-Pourquoi ne m'apportez-vous pas un vêtement ordinaire?

- Vous voyez ma tenue?

- Ce n'est pas mal. C'est même élégant.

- C'est un hologramme... (Nothomb, Péplum: 19).

A côté des informations qui permettent au lecteur de s'informer et de comprendre le cadre du dialogue (les interlocuteurs, le temps et l'espace), nous remarquons également que les paroles de l'écrivaine et de Celsius décrivent successivement un lieu plus large et plus grand que la basilique: c'est le vingt-sixième siècle.

Egalement, d'autres informations dans le dialogue parlent de la suppression des vêtements, de l'extinction des ruminements,

(La stratégie narrative ...) Dr. Alaaedin Baheidin Alaaedin 
du Grand Dépôt, de la prolifération des musées, des crises énergétiques des nouvelles formes d'énergie, et de l'anéantissement du Sud...etc., tous ces événements ne sont pas décrits en détails, mais nous avons l'impression que chacun insiste sur un détail de manière caricaturale:

"-Voyons... En 2248, on a découvert que le safran avait des propriétés souveraines dans le traitement des maladies mentales.

-Poétique. Continuez.

-La crise de l'énergie a entraîné, dès le début du vingt-deuxième siècle, des bouleversements politiques considérables.

-N'importe qui aurait pu le prévoir.

-En réaction contre ces mutations, l'Académie française a précédé à un suicide collectif..." (Nothomb, Péplum: 87).

Pour l'écrivaine séquestrée, il est trop difficile d'avoir une preuve véritable qu'elle est bien et belle en 2580 et qu'il ne s'agit pas d'une farce; elle ne croit que la parole de Celsius. Pour les mêmes causes, il est difficile pour le lecteur d'avoir une preuve valable ou de s'imaginer avec véracité le cadre le plus large du dialogue, c'est pourquoi, la romancière évite une description exacte du futur qui donne au lecteur la liberté de se figurer l'avenir à l'aide de quelques détails évocateurs.

\section{2) La fonction dramatique}

Il est à remarquer que dans les romans dialogués de Nothomb, les intrigues prennent la forme de situations conversationnelles qui ne sont, en fait, que des affrontements ou

(La stratégie narrative ...) Dr. Alaaedin Baheidin Alaaedin 
des confrontations car: "le dialogue est le développement, la mise en forme de deux positions discursives confrontées ou affrontées." (Ubersfield, 1993: 263), et cela se manifeste clairement dans "Péplum" qui: "est construit selon le mode du dialogue, forme de récit préféré par Amélie Nothomb." (Cottille-Foley, in Chimères, 2003: 48). La conversation dans le roman "Péplum" se transforme rapidement en un duel verbal où: "les interlocuteurs ont des vues, des sentiments, ou de passions qui se combattent." (Marmontel, 1968: 381).

Ce genre de dialogue est la forme requise quand nous cherchons à produire des effets dramatiques: "La polémique est un catalyseur d'action, qui participe souvent d'enchaînement narratif codés." (Adam et Durrer, 2001: 169). La fin heureuse de "Péplum" représente une exception, à la fin de ce roman, nous apercevons que Celsius et A.N. s'insultent et se battent au moment où: "le dialogue est là pour faire avancer l'action en instituant un projet, voire une tension." (Durrer, 1990: 118).

Les scènes d'affrontement entre les personnages qui ont lieu à la fin de "Péplum", contribuent à faire les événements, car les personnages dans ce type de dialogue tentent d'en venir à un accord pour que la situation change, sans quoi l'échange se termine par le meurtre. D'autre part, ces scènes d'affrontement à la fin du roman aident également à faire l'action et par conséquent, faire progresser le savoir des personnages et celui des lecteurs. 


\section{3) La fonction psychologique}

Ce qui manque aux lecteurs des romans dialogués d'Amélie Nothomb c'est qu'ils ne connaissent pas trop l'apparence des personnages dans ces romans. Ici, vient le rôle indispensable $\mathrm{du}$ dialogue romanesque qui peut combler ce manque de connaissance en occupant une fonction psychologique qui s'intéresse avant tout par l'analyse psychologique des caractères dans les romans, ainsi que leurs impressions et leurs objectifs:

"La conversation porte davantage sur la psychologie des personnages que sur leur apparence extérieure ou sur leur situation sociale et ils semblent perdre peu à peu de leur matérialité." (Moline et Lafhail- Moline, 2003: 167).

Dans "Péplum", les sentiments intérieurs des personnages se montrent à travers leurs paroles. Nous apercevons dans le roman deux interlocuteurs qui se montrent impudents et se trouvent haïssables. Mais, quand la question concerne l'amour, ils deviennent plus tendres et plus humains et cela se manifeste lorsque A.N. pleure lorsque Celsius dit que ceux qu'elle aime sont morts il y a longtemps, tandis que Celsius se montre sensible lorsqu'il se souvient de Pompéi :

"Comme elle était belle, la ville que j'aimais: Tellement plus belle qu'à votre époque, où tant d'archéologues amateurs l'avaient écorchée!..." (Nothomb, Péplum: 138). 


\section{Conclusion}

L'œuvre d'Amélie Nothomb représente l'illustration de l'image japonaise dans la littérature française contemporaine, car le Japon représente un sujet inépuisable dans l'œuvre de Nothomb. Son caractère est japonais mais elle reste un miracle belge. Elle est une auteure belge de langue française, son œuvre est traduite dans le monde entier.

Dans la de notre travail, nous avons mis la lumière sur les caractéristiques de l'écriture de Nothomb, celle-ci pense que son écriture n'est qu'une simple définition du monde et du langage en essayant de donner les noms justes aux choses.

Nous avons abordé également les thèmes prédominants traités par Nothomb dans son œuvre comme la solitude qui se trouve dans presque la majorité de ses romans. Elle croit qu'il est important de faire une distinction entre la solitude qu'elle avait subie et la solitude idéale qui se manifeste clairement dans son roman "Les Catilinaires", où nous trouvons la solitude qui offre le calme tellement désiré en refusant l'existence humaine. Le deuxième sujet traité par Nothomb, c'est le thème du corps humain. Nothomb s'intéresse dans ses romans au corps humain, car ce corps représente le révélateur de l'identité, elle a proposé ce corps avec ses deux faces: le corps beau et le corps difforme. Quant au troisième sujet, c'est le thème de la mort avec ses deux genres: la mort réelle et la mort imaginaire qui se manifeste également dans son roman "Les Catilinaures".

(La stratégie narrative ...) Dr. Alaaedin Baheidin Alaaedin 
Egalement, dans notre étude, nous avons analysé les techniques langagières de Nothomb en parlant du pouvoir du langage et son importance, qui aide à développer les réflexions et comprendre le sujet. Le langage nothombien se caractérise par sa réalité qui est la plus importante dans l'écriture de notre écrivaine et qui l'aide à exprimer ses impressions, et cela se manifeste dans son roman "Les Catilinaires".

Nous avons abordé le thème du roman dialogué de Nothomb. Nous pouvons dire que le roman "Péplum" est un roman dialogué par excellence, dans lequel, nous avons étudié la narration dialoguée. La parole des personnages dans ce roman se caractérise par la dramatisation où l'écrivaine a réussi à mettre en évidence les échanges verbaux où la parole joue un rôle dans le développement de l'intrigue et de l'action.

A la fin de notre étude, nous avons étudié les différentes fonctions de la narration dialoguée ou conversationnelle dans le roman "Péplum", en commençant par la fonction descriptive et son rôle dans la représentation de l'action et des personnages, ensuite, la fonction dramatique où les intrigues prennent la forme des situations d'affrontement et de confrontation. Et enfin, nous avons traité la fonction psychologique qui aide à mieux connaître les personnages des romans nothombiens. 


\section{Bibliographie}

\section{I- Corpus}

1- NOTHOMB Amélie, Les Catilinaires, Paris, Albin Michel, 1995.

2- NOTHOMB Amélie, Péplum, Paris, Albin Michel, 1996.

\section{II- Ouvrages généraux}

1- ADAM Jean-Michel et DURRER Sylvie, Conversation et dialogue, Paris, Albin Michel, 2001.

2- BERTHELOT Francis, Paroles et dialogue dans le roman, Paris, Nathan, 2001.

3- BLANCHOT Maurice, La douleur du logique, in Le livre à venir, Paris, Gallimard, 1971.

4- BOBLET Marie-Hélène, Le roman dialogué après 1950. Poétique de l'hybridité, Paris, Honoré Champion, 2003.

5- BOURNEUF Roland et OUELLET Réal, L'univers $d u$ roman, Presses Universitaires de France, 1989.

6- GENETTE Gérard, Figure III, Paris, Seuil, 1972.

7- GUELLOUZ Suzanne, Le dialogue, Paris, PUF, 1992.

8-MARMONTEL Jean-François, Eléments de littérature, Euvres complètes, tome IV, $1^{\text {er }}$ partie, Genève, Slatkine Reprints, 1968.

9-MOLINE Jean et LAFHAIL-MOLINE Raphaël, $\underline{\text { Homo }}$ fabulator, Théorie et analyse du récit, Montréal/Paris, Leméac/Acte sud, 2003.

10-MYLNE Vivienne G., Le Dialogue dans le roman français de Sorel à Sarraute, Paris, Univeritas, 1994.

11- UBERSFIELD Anne, Lire le théâtre, Paris, Editions sociales, 1993.

\section{III- Ouvrages consacrés à Nothomb et son œuvre}

(La stratégie narrative ...) Dr. Alaaedin Baheidin Alaaedin 
1- AMANIEUX Laureline, Amélie Nothomb, l'éternelle affamée, Paris, Albin Michel, 2005.

2- DAVID Michel, Amélie Nothomb, l'écriture illimitée, Paris, Harmattan, 2013.

3- DE DECKER, Jacques, Préface: Nothomb avec un $B$ comme Belgique, in Susan Bainbrigge et Jeanette Toonder, Amélie Nothomb, Authorship, Identity and Narrative Practce, New York, Peter Lang, 2003.

4- KOBIALKA Margaux, La création d'Amélie Nothomb à travers la psychanalyse, Paris, Editions Le Manuscrit, 2006. 5- NARJOUX Cécile, Etude sur Stupeur et tremblements d'Amélie Nothomb, Paris, Ellipses Editions Marketing, S.A., 2004.

6- ZUMKIR Michel, Amélie Nothomb de A à Z: portrait d'un monstre littéraire, Bruxelle, Editions le grand miroir, 2003.

\section{IV- Articles de revues portant sur Amélie Nothomb}

1- DELANGUE Henri, "Autobiographie ou autofiction chez Amélie Nothomb?", in Cédille, No. 10, avril 2014, pp. 129141.

2- GINGRAS Chantale, "Amélie Nothomb: Survol d'un oiseau rare", in Erudit, No. 128, 2003, pp. 37- 40.

3- NUSRAT Ahmad, Amélie Nothomb. Ange ou démon? In Sensa, 2001.

4- SAUNIER Emilie, "Accéder à la reconnaissance en tant que femme écrivain belge: Une étude du cas d'Amélie Nothomb dans le champs littéraire français", in Erudit, vol. 47, No. 2, 2015, pp. 113- 135.

5- VIGEANT Louisz, "Amélie Nothomb, Antigone ou

Cassandre", in Erudit, No. 83, 1997, pp. 162- 165.

6- WILWERTH Evelyne, "Amélie Nothomb sous le signe de cinglant", in La Revue Générale, No. 6-7, 1997.

(La stratégie narrative ...) Dr. Alaaedin Baheidin Alaaedin 


\section{V- Articles de revues généraux}

1-COTTILLE-FOLEY Nora,"Abomination et Sacralisation dans Hygiène de l'assassin", in Chimères, 2003, vol. XXVII, pp. 47- 57.

2- DURRER Sylvie, "Le dialogue romanesque, essai de typologie", in Pratiques, No. 65, mars 1990, pp. 37- 62.

3-MITTERAND Henri, "Dialogue et littéralité romanesque", in Le Dialogue, Ottawa, Marcel Didier, 1985.

\section{VI- Interviews}

1- Interview avec l'auteure, le 12 mai 2009, chez son éditeur Albin Michel.

2- Interview avec l'auteure, le 22 mars 2010, chez son éditeur Albin Michel. 


\section{ملخص}

ولدت الكاتبة إميلي نوتومب في اليابان في سنة لو 19 ـ لقد ظهرت اليابان في أعمال نوتومب كثئ روائي هام. فالتأثير الأدبي لليابان في أعمالها كان واضحا للغاية في قصصها الخيالية وكذلك في رواياتها التي تحكي سيرتها الذاتية. فمن خـلال دراستتا، سوف نلقي الضـوء على التقنيـة الكتابيـة للكاتبـة نوتومب والدور الذي لعبته في العلاقات الإنسـانية كذلك الموضـوعات التي سـيطرت وشـلت عقل نوتومـب. فروايـة "الخطابـات المعادة" سنشكل أسـاس الدراسـة في الجزء الأول من الدراسة، أما في الجزء الثاني من دراستنا، فسوف نقوم بمعالجة الوظـائف المختلفـة للسـرد الروائي لنوتومـب مـن خـلال روايـة "المشـمل"، مثل الوظيفة الدرامية والوظيفة النفسية وكذلك الوظيفة الوصفية. كلمات مفتاحية: استراتيجية- رواية- إميلي نوتومب 